Infusionstherapie 1991;18:262-263

\title{
MEDICA Program
}

MEDICA'91 20. bis 23. November 1991 - Düsseldorf - Messegelände Kurzprogramm

9.15

Mittwoch, 20. November 1991

12.30 Uhr 14.30-18.00 Uhr

Donnerstag, 21. November 1991

\subsection{5-12.30 Uhr 14.30-18.00 Uhr}

So hographíe des Abdomens -Grundkurs

Praxisbezogenes Seminar mit Fallvoretellungenanhand von Videobändern und Diapositiven

Prof. Dr. E. G. Loch, Wiesbaden, Prof, Dr. R. Heckema円n, Bochum

So \ographisches Praktikum für Fortgeschrittene -

Praxisbezogenes Seminar mit Fallvorstellungen a $\pi$ hand von Videobändern und Diapositiven

Prof. Dr. E.-G. Loch, Prof. Dr. P. Linhart, Dr. J. A. Bönhof, Wiesbaden

InterdisziplinäresFortbildungsforum der NordrheinischenAkademie für ärztll. Fort-und

Weiterbildung(Fortsetzung

Freitag/Samstag). Die Vort rage

werde $\cap$ vomRegierungspräsidentDüss.alsAusbildungsveranstaltu $\mathrm{n}$ für AiP

anerkannt.Forder@SiedasausführlicheProgrammanÎAdressesiehegegenüberFreitag/Samstag, Rau $\mathrm{mlO})$ :

Prof.Dr.W.Wildmeister,Kempen 36 I

BronchologischerUntersuchungskurs -Fortsetzung

Prof. Dr.J.A. Nakhosteen, Bochum und Dr. D. Greschuchna, Essen

BronchologischerUntersuchungskurs(Phantomkurs)

Prof. Dr.J.A. Nakhosteen, Bochum und Dr. D.Greschuchna, Essen

$\mathrm{XJ}$ CO

"c

$\mathrm{CD}$

Ç»cõ

CO Çü

$\sigma)$

$\mathrm{cz}$

2

ò

$\mathrm{CO} \mathrm{CO}$

$\varphi$

o

Hochdruck und Begleiterkranku ge $\pi$

Prof. Dr. K. Hayduk, Düsseldorf, Prof. Dr.Th. Philipp, Essen; 
Veranst.:Dt.Hochdruckligae.V. 2

Notfallmedizin im Alter - Möglichkeite $\bigcap$ und Grenzen

praktischenHandelnseinschlíefilichlntensivmedizin

Prof. Dr. med.habiI.O. Barrels, Nümberg 3

Selen -einessentiellesSpurenelementinderDiskussion

Prof. Dr. G. N.Schrauzer, San Diego/USA 4

MEDICAiuristica

Rechtsf ragen der ärztlichen Schweigepf licht

RAM.Broglie, Wiesbaden

Gesundheitspolitische Podiumsdiskussion

Prof. Dr. F. E.Münnich, Bonn Veranst.:MPS

Begleíterkrankungen und Spätfolge $\bigcap$ bei Diabetes mellítus

Dr. H.J.Rülimann,Dínslaken

Schilddrüsen-Seminar

Prof. Dr. F. A. Horster, Düsseldorf

Sinnesorganeim Alter

Prof. Dr, K.-F. Hamanก, München

Ambula $\cap$ teChirurgie und invasive ambulante Diagnostik

Dr. E.Eicher, Basel

FrüherkennungundFrühtherapiederpostmenopausale

Osteoporose

Prof.Dr.J.D.Ringe,Leverkuse $\quad 16$

NeueAspektebeichronische $\cap e \pi t z u ̈ n d l i c h e \pi$

Darmerkrankungen

Prof. Dr. H.Goebell, Essen

PharmakotherapeutischeSchwierigkeiten in der Praxis

Prof. Dr. Ellen Weber, Heidelberg 1

MEDICAiuristica

DerKassenarztimSpa nungsverhältniszwischen

Wirtschaftlichkeitsa $\pi$ gebot und Regeln der ärztlichen Kunst

RíchterG.Schroeder-Printzen, Kassel 19

ngunge?

Enlzündu gsbestrahlu $\mathrm{n}$ u ter amBewegungsapparat

Prof. Dr. A. Klümper, Freiburg

Workshop Hyperinsulinämíe -insulinresistenz und Hyperinsulinämie -

KonsequenzenfürdieTherapie

Prof. Dr.W. Krone, Hamburg

NaturheilkundeundSchulmedizin

Prof. Dr. Dr. h.c. Hildegard Wagner, Müncten

Honorarkürzu genundArzneimittelregresse

RAM.Broglie, Wiesbaden

Veranst.: Inst. für Arzt · und Kassenarztrecht

Wenn die Seele streikt und der Körper antwortet

Psychosomatik in der Hausarztpraxis

Dr. G. Gerhardt, Wendelsheim

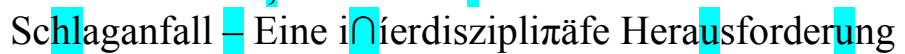


- Forum furÄГZte -

Prof. Dr.W. Hacke, Heidelberg 28

EKG·GrundkursTeill Prof. Dr. E. G rosser, Krefeld

Laser in der Medizin - Minimal Invasive Chírurgíe/

Zahnmedizin

Prof. Dr. R.Steiner,Ulm

24-Stunden-Blutdruckmessung Prof. Dr. B.Krönig, Trier

Veranst.:Dt.LigazurBekämpfügdeshohen Blutdruckse.V.

Peripherearterille Durchblutungsstörungen

beimjungenMenschen

32

Prof. Dr. H. Rieger, Engelskirchen

Novellierung des GRG und europäischePerspektiven

- Pressegespräch-

Dr.H.vonLoeper, Neuss

Veranst.: BPI 33

AktuelleKonzeptederVarizentherapie Dr. E.Rabe,Bonn

Diagnostik der Hepatitis C

35

Prof. Dr.|P. Küh円1, Hamburg PDDr.M.Köhler,Homburg/Saar

SchicksalsschiagSch aganfall?Gesundheitsforumfürdie

Praxis - Forum fürÄrzte, Journalisten und Betroffene -

Prof. Dr. H.-C.Diener, Essen 28b

EKG-GrundkursTeíl2 Prof. Dr. E. Grosser, Krefeld

AtemphysiotherapieundKrankengym astik

inderPneumologie

Prof.Dr.U.H.Cegla,BadEms

24-Stunde $\cap$ Blutdruckmessu $\pi \mathrm{g}-$ Wiederholung

Prof. Dr. B.Krönig, Trier

Veranst.:Dt.LigazurBekämpfung des hohen

Blutdruckse.V.

Die \eue Art des ärztlichen Marketing für Praxis-Teams

Prof. Dr. G. F. Riegl, Augsburg

Veranst.: Insl. f. Management im Gesundheitsdienst,

Augsburg

Orie`tierungs- und Nachschulu gskurs

- Die korrekte Abrechnung - Dr. H. Massing, Ibbenbüren Veranst.:[BPA

BehandlungskonzeptederPsychosomatische $\pi$ Medizin

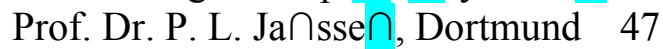

PhysikalischeMedizin Prof. Dr. J. Kleditzsch, Pirna

Der Patient und seinArzt -Die Sprache der Krankheit

Dr. med. W. Pícker-Huchzermeyer, Bad Saizuflen und Dr. phil. R. Drommel, Forst

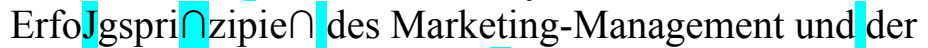

Corporate Identity im Kranke $\pi$ haus

Prof. Dr. G.F. Riegl, Augsburg 
Veranst.: Instituî für Management im

Gesundheitsdienst, Augsburg 37

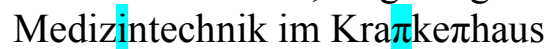

Dipl.-lng.G.Lehma $\cap$, Bielefeld

Veranst.: Arbeitskreis Medizintechnik der FKT

Planung, Organisation, Kosteneinsparung inArztpraxen

0 . C.Trillinger, Ohlsbach

VerschläftdasPharma-MarketingdenParadigmenwandel

inderMedizin?

Dr. R.-E. Hoch, Sprendlingen

Veranst.: BPA 38

Neue Wege in der Prävention in der Kassenpraxis

Dr. M. Klein-Lange, Oldenburg Veranst.: KV Niedersachse

lOJahreWirkprinzipACE-Hemmung

- GegenwärtigerStand und Zuku $\cap$ ftsperspektiven -

Prof. Dr. V. Heimsoth, Damp

Die Prävention der kardiovaskuláre $\cap$ Risíkofaktoren - eíne bevölkerungsmedizinische und gesundheitspolitische Aufgabe

Prof. Dr. U. Keil, Bochum, Prof. Dr. D. Klaus, Heidelberg Veranst.: Dt. Hochdruckltgae.V.

Allergologische in vitro Diagnostik

Prof. Dr. G. Forck, Münster

Veranst.: Ärzteverband Deutscher Allergologen e.V.

Der depressive Alterspatient Prof. Dr. H.·J.Möller, Bonn

DarmflorainSymbioseundPathogenität Prof. Dr. Dr. L Demling, Schlüsselfeld

ÜbungenzurLunge $\pi$ funktionsdiag $\cap$ ostikfürArzthelferinnen

Dr.W. Fleischer, Ingelheim 40

Pneumologie '91: Therapie von Asthma und COPD Prof. Dr, D. Nolte, Bad Reíche hhall

Universitäts-Frauenklinik Düsseldorf

Uitraschall in Gynäkologte undGeburtshilfel

Einführungskurs mit Patientendemonstrationen PDDr. Peter Kozlowski, Düsseldorf

Universitäts-Frauenklinik Düsseldorf

Uitraschall in Gynäkologie uกd Geburtshilfell Prä hatale Diagnostik undTherapie(Video-

Seminar) PD Dr. Peter Kozlowski, Düsseldorf

Eingang Nord/Halic 6/1, OG

Kolposkopiekurs(Basiskolposkopíe)

Einführung indie Kolposkopie, normale und abnormekolposkopische Befundean Portio und Vagina

Dr. St. Seidl, Hamburg, Dr. P. Schomann, Lüneburg

EDIC A informatica

Gut informiert - besser heilen

205

H.Schultze, MEDISOFT, Frankfurt 
GrundkursA

Ubungen am Praxis·Computer-System MeDO-med Grundkurs füralle, die nochkeine EDV haben, aberwissen möchten, was EDV in der Praxis leistet

Dr. med. W.Hüttemann, Aachen

Dipl.-Ing,Susan $\cap$ eHütteman $\pi$, Aachen 206

MEDICA informatica

Medizinische Expertensystemeam Beispielder

Schilddrüse $\pi$ diagnostik

I $\pi$ vestitions-und Kostenberatung derZahnärzte

vorderNiederlassung

Dipl.-Volksw. P. J. Müller, Kõln

Veranst.: Institut der Dt.Zahกärzte 210

Dr. M.Zügel, Mannheim

GrundkursA

- Wiederholung der Veranstaltu $\mathrm{g}$ vomVormittag-

Grundkurs B

Ubungen am Praxis-Computer-System MeDO-med

Grundkurs fürArzthelferinten, die keine EDV haben,

sieabergernei ihrerPraxisorganisationhätten

Dr. med. W.Hüttemann, Aachen Dipl.-ing.SusanneHüttemann, Aachen

211

Dr. med. W.Hüttemann, Aachen Dipl.-lng.SusanneHüttemann, Aachen

MEDICA informatica

Einführung in die labormedizinischeWissensverarbeitung amBeispielderExpertensystem-

SchaleProM.D.

Prof. Dr. Chr.Trendelenburg, Frankfurt

AufbaukursC

Übungenam Praxis-Computer-System MeDO-med

Aufbaukurs für alle, dieden Grundkurs A oder Bbesucht

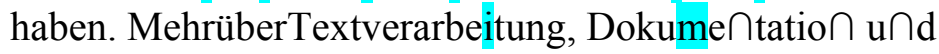

Abrechnung

Dr. med. W.Hüttemann, Aachen

Dipl.-lng.SusanneHüttema nn, Aachen

223

Praxis-Controlling zurwirtschaftiichenPraxisführung - mitundoh $\cap e P C$

Dipl.-Kfm. 0. Henker, Reutlingen

EDVinderArztpraxisamBeispielSISYMEDansparenz,mehrEffizie $\cap z$, mehrSicherheit!

Dr.K. König, Steinbach/Taunus

Dr. J. Moeller, Lampertheim 212

Workshop:|»lníegrierteTextverarbeitung« Demonstration

derLeistungsfähigkeitu terschiedlicherPraxiscomputer

Gudrun Demnick-Klas, Wöllstadt

D.Fricke, Hannover 219

Das reiatio naleDatenbanksystem ORACLE in der

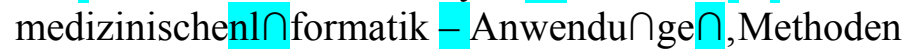

undVerfahren 
Dr.G. H. Himmelman n, Marburg 224

MKC (Messe-Kongress-Center\} Ost

$\mathrm{CO}$

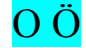

E. Göckeler-Leopoid, Soest

BIOTEC

Cytokine:RegulatormolekülederZellproliferationund

-differenzierung

Prof. Dr. M. F Rajewski, Essen 301

HautundUmwelt

Prof.DrDr.J.Ring, Hamburg

DieManager-Konferenz - Erfolgsplanung, Erfolgskontrolle, Steuerstrategie $\pi$ Dr.G.Schlicht,

Mannheim Dipl.-Kfm. M.Bender,Jülich Veranst. :AKAMEDMa $\cap \cap$ heimGmbH

smetabolischeSyndrom

Prof. Dr. U. Klör, Gieíien

AllergieundUmwelt

Prof. Dr.Dr.J.Ring, Hamburg,

Prof. Dr. H. Behrendt, Düsseldorf

Veranst.: Dt. Ges. f. Allergie-u. Immu $\cap$ forschu $\cap g$

ModemeAspektederzellvermitteltenundhumoralen immunantwort

PDDr.F.E.Krapf,Erlangen

BIOTEC

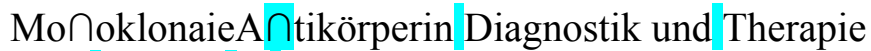

Prof. Dr. H.Sies, Düsseldorf

Akupunkturi门derOrthopädieundSportmedizin Dr. A. Molsberger, Düsseldorf, Dr. G. Stux,

Düsseldorf

DieHonorar-Abrech $\cap u n g$

Dipl.-Volksw. D. Jollmann, Hamburg Veranst.: AKAMED Mannheim GmbH

$1 \cap$ fektio $\cap e \cap$ in der Praxis

MEDIC A'91 20. bis 23. November 1991 - Düsseldorf - Messegelände Kurzprogramm

Freitag, 22. November 1091

9.15-12.30 Uhr 14.30-18.00 Uhr

Samstag, 23. November 1991

9.13-12.30 Uhr $\quad 14.30-18.00 \mathrm{Uhr}$

Arzt im Rettungsdienst - Teil B

Dr. H. E. Purrmann, Wuppertal, Prof. Dr. G. H, Engelhardt, Wuppertal

Vera $\pi$ st.: NordrheinischeAkademiefürärzíl. Fort-u.WeiterbildungderÄKNordrhein

Arzt im Rettungsdie $\cap s t-$ Teil B - Fortsetzu 9

Dr. H. E. Purrmann, Wuppertal, Prof. Dr. G. H. Engelhardt, Wuppertal

Vera円st.: NordrheinischeAkademiefürärztl. Fort-u. WeiterbildungderÄKNordrhein

LaboГdiagnosiikinderprä-und perinatalen Medizin - $\not \mathrm{k}$ )ein Vergleich mit bildgebenden Verfahren?

Prof. Dr. E.-G. Loch, Wiesbaden 
Die einf ache Lu@ge $\cap$ f unktionsdiag $\pi$ ostik fürArzte -

TheorieundpraktischeÜbungenfürÄrzîemitVorke $\cap n t n i s s e n$

Dr.H.Físcher,Weilheím

55

Prof. Dr. F. Deinhardt, Münche $\pi$

ModerneArrhythmiebehandlung

Prof. Dr. B. Lüderitz, Böí

NeueAspektezurTherapiederKHK Prof. Dr. P. Hanrath, Aachen

Internistischelntensivmedizin

Prof. Dr.A. Sturm, Herne

Reflextherapien bei Erkrankungen des

Bewegungsapparates

Univ.-Doz.Dr.M.Eder,Graz

Dr. K.-H. Bergis, Bad Merge theim

Perspektivendermedikamentösen Oifferentialtherapie

mitKalziuma $\operatorname{tagonisten}$

Katastrophenmanagement im Krankenhaus Prof.Dr.P.Sefrin, Würzburg

Dipl.-Kfm. 0. Henker, Reutlingen

GrundkursD

Übungen am Praxis $\cdot$ Computer-System MeDO-med

Grundkursvon der Einführung in die EDVbiszuhaus-L

fachärztlicherVersorgung

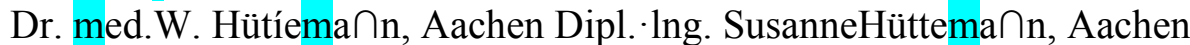

Klinische Prüfu $\mathrm{g}$ im politischen Umfeld

-Überwachung,Aufklärung,Verantwortlichkeiten-

Prof. Dr. UrsulaGundert-Remy, Berlin

Dr. Cl. Kori-Lind ner, Nürnberg; Veranst.: FÁPI

- $\sigma$ o $\mathrm{C} \Lambda$

$\phi \mathrm{D}$

-a. $\omega \mathrm{O}$

$\sigma>\sigma>\omega$

$\sigma)$

$\mathrm{O}$

ஸ́

$\mathrm{CO}$

$\mathrm{CO} C D$

ü

o o

CO "Õ5

I

CD

$\mathrm{E}=\mathrm{CO} C \mathrm{CD}$

$\mathrm{Lu}$

CO

o ó 
Angiologie in der Praxis:

Dífferentialdíag nostischeunddifferentialtherapeu $\propto$ ische

Problemederpheripher-arterielienVerschlufikra $\cap$ kheit

Prof. Dr. H.Heidrich, Berlin 79

Hochdruck:

Ein Stoffwechsel-Syndrom?

Die Kompressio $\pi$ stherapiebei derchro $\pi$ ischen

venösenlnsuffizie $\cap z$

Auge erkranku $\pi \mathrm{ge} \cap$ inderPraxisdesniedergelasse $\pi$ en

Arztes

Prof. Dr. K. W. Ruprecht, Homburg

Veranst.:Berufsverb.derAugenärzte

Deutschla $d$ dse.V.

61

Prof. Dr. G.J.Dietze, Bühl

Prof. Prof. h. c. Dr. Dr. h. c. N. Klüken, Essen

Diagnostik von Probieminfektionen

Strahle $\cap$ risíko inderrontge $\pi$-u. nuklearmedizinischen

Diagnostik?

Prof. Dr. Chr. Reíners, Essen

Vera $\cap$ st.:Verei $\pi$ igu $\pi$ gDeutscher

Strahlenschutzärztee.V.

Haftungsrisiko der MTA

FamiliäreAde กomatösePolyposis \{FAP)

DrastischePrognosebesserungdurchkonsequentes

Familienscree ing

Gertraud Schmidt, Lemgo

Veranst.:dvta

Prof. Dr. Ch.Herfart i, Heidelberg

Prof. Dr. H. Buhr, Heidelberg

Dr.Dr.Th.Fenner, Sölden

Diabetes - neuediagnostischeundtherapeutischeAkzente

Die Hämodilution als therapeut $1 / 8$ sches Prinzip bei

periphere $\cap$ arteriellen Verschlußkrankheiten und cerebralen Durchblutungsstörungen

- Infusío กstherapie In Klinik und Praxis -

Prof. Dr. H.Böhme, Gauting

Derl $\pi$ sulin-behandelte Diabetes mellitus

Das ersteJahr in freier Praxis-Erfahrungen,

Erkennt $\pi$ isse - Hinweise und A $\cap$ regungenfür

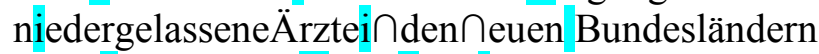

und seiche, diees werden wolle $\pi$

D.Bahnemann,Kóln

Veranst.: VerbandWirtschaft u.Arzíe.V.

92

Prof. Dr. F. A. Gries, Düsseldorf

“

Mykoiogie:NeueTherapiemòglichkeiten

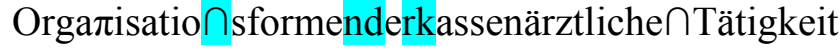


unter Befücksichtigung der Versorgungsstrukture $\pi$

in den 5 neuen Bundesländern

Dr.iur.R.Hess,Köln

Veranst.:KBV 71

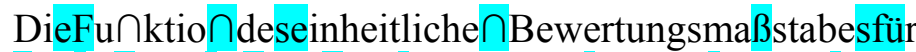

arztliche Leistungen als Instrument zurGestaltu $\cap \mathrm{g}$ der

kassenärztlichenVersorgu $\mathrm{g}$

Dr.M.Moewes, Kõln

Vera $n$ st.: KBV $\quad 82$

Prof. Dr. R.Gotzen, Berlin

Prof. Dr. W. Meinhof, Aachen

Notfallmedíkamente -medikamentöseNotfalltherapie

Was íchschon immerwisse $\pi$ wollte

Prof.Dr.P.Sefrin, Würzburg 7i

Differentialdiagnoseu.therapeutischeMöglichkeitenbei

Hirnleistungsstörungenim Alter

Prof. Dr.G.Paal,München 83

Prof. Dr. H.-J. Vogt, München

Die Rolleder MineralsioffeundSpurenelementeinder Ertahrung

Prof. Dr. J. D. Kruse-Jarres, Stuttgart

Prävention in derárztlichen Praxis KarinDiehl, Dortmund Veranst.: BdA

Arzt und öffentliche Meinung

Sínd die Mediziner richtis informiert?

K.Dallibor, Offenbach

Holekuiarbiologische Diagnostik in derTransfusionsmedizin

Prof.Dr.S.Seidl, Frankfurt 84

Ì1

Interdisziplínäres Fortbildungsforum der NordrheinischenAkademiefür arztliche Fort- und

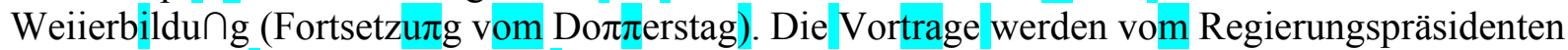

Düss. als Ausbiîdungsvera $\cap$ staltui

Forder $\pi$ SiedasausführlicheProgramman:Nordrhein|scheAkademie ${ }^{\wedge}$

Prof. Dr.W.Wildmeister, Kempe 62 I

BronchoiogischerUntersuchungskurs - Fortsetzung

Prof. Dr. J. A. Nakhosteen, Bochum und Dr. D. Greschuchna, Essen

BronchologischerUntersuchungskurs-Fortsetzung

Prof. Dr. J. A. Nakhosteen, Bochum und Dr. D. Greschuc.h円a, Essen

Farbdoppler-Echokardiographie-Kurs

Prof. Dr. H. Gülker und PD Dr. J. Thale, Wuppertal

Farbdoppler-Echokardiographie-Kurs - Fortsetzung Prof. Dr. H.Gülker und PD Dr. J.Thale,

Wuppertal

Praktische Kolo-Proktologie Dr.J.J.Kirsch, Mannheim Veranst.: Berufsverband

Umweltmedizin GesundheitundUmwelt Prof. Dr. H.Eckel, Hannover

Gynäkologische Hormontherapie: Eine aktuelle Praxisorientierung Prof. Dr. E.-G. Loch,

Wiesbaden

ModerneVerba\dstech $\pi$ ik mit praktischen Ubungen

Prof. Dr. med. habil. K. Steinbrück, Stuttgart97 
Diagnostik und Therapie akuter und chronischer Kreuz-und Beinschmerze $\pi$ (1schialgie)

Prof. Dr. med.habil.J.Jörg, Wuppertal

Ergometrie-Seminar ' 91

Prof. Dr. H. Löilge@, Remscheid

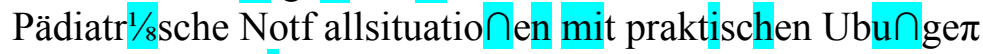

Dr.A. Dorsch, Sigmertshausen 76

Herzrhyth $\pi \cap$ usstöru gen im Notarztdienst - Analyse und

therapeutische Intervention mit praktischen Übungen

Dr. A. Dorsch, Sigmertshause 98

Eingai \ð Nord I Halle 6/1. OG

Kolposkopiekurs II (Fortgeschrittene) - Wiederholung des Do円nerstag-Kurses

Dr.St.Seidl,Hamburg, Dr. P. Schomanก,Lüneburg

237

Kolposkopiekurs I (Einführung)-Wiederholung des Mittwoch-Kur; Dr.St.Seidl, Hamburg, Dr. P. Schumann, Lüneburg

KoloskopischerUntersuchungskurs - Fortsetzung Prof. Dr. U. von Gaisberg u. Mitarbeiter,

Stuttgart

MyofascialeTriggerpunkte, Akupunkturund Kranken-

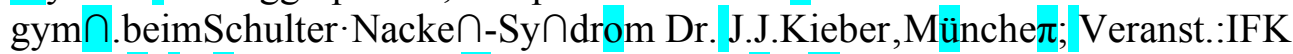

Koloskopischer Untersuchu@gskurs mit Videodemonstraiion und Ubungen am Phantom

(Stuttgarter Koloskopiekurs)

Prof. Dr. U. von Gaisberg und Mitarbeiter, Stuttgart 22

Hämatologiekurs für Fortgeschrittene - Leukämie-Diag nostik (Beurterlung von Blut- und

Knochenmarkausstrichen einschl.Zytochemie)

Prof. Dr. P. Lorbacher, Wiesbaden

Hämatologiekurs/MikroskopierkursfürMTA Prof. Dr. K. P. Hellriegel, Berlin

informat io \skurs für manuelleMedizin - Fortsetzung

Dr.G.Marx,Hittenkirchen

Veranst.: DeutscheGesellschaft für manuelle Medizine. V.

Informationskurs für manueMe Medïzin

Dr.G.Marx,Hittenkírchen

Veranst.: Deutsche Gesellschaft $\mathrm{f}$ ürmanuelle Medizine. V.

KBV-PläneundanderekünftigeRahmenbedingungen fürdas Labor - Labor-Management-Forum

MedizinischePhotographieundBildverarbeitung

Dr. H. Gu ddlach, Oberkochen

Chance fürlhrePla@ung, Organisation und Koste`.

mínimíerungeinerma nuellen Organisation auf eine

EDV-Aufbau-und-Ablauf-Orga Пisation

O.CTrillinger, Ohlsbach 233

MEDICA informatica

MEDICA informatica

MEDICA í $\pi$ formatica

MEDICA informatica

MEDICA informatica

perimed-lnformations $\cdot$ Seminar AnforderungenaneinArztcorriputersystemder90erJahre 
EínschätzungdesEDV-Eiกsatzesinder

ambulanten Medizin vom Standpunkt der

Selbstverwaltu $\pi \mathrm{g}$

Dr. E. Geiєs, Köln; Veranst.:ZI

Pro und Contra für die EDV in derärztlichen Praxis mitpraktischen Beispielenam PC

Ozontherapie

Prof. Dr. S. Rilíing, Stutigart

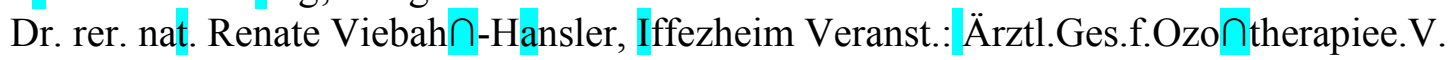

N.N.

- $1 . \cdot$

J. Köferl, ELM ED SYSTEMS, Nagel

AufbaukursE

Übungen am Praxis-Computer-System MeDO-med

Aufbaukursfüralle, diede $\cap$ GrundkursAoderBbesucht

haben.MehrüberintegriertesArzneimiîtel-1 $\cap$ formations-

System und Praxis-Marketing

Dr. med. W. Hüttema $\cap$, Aachen

Dipl.-lng. SusanneHüttema nn, Aachen 235

Spezialkurs F - Teil $1+2$

Übungen am Praxis-Computer-System MeDO-med, Kurs für alle, die nochkeine EDV haben und

siesich in ihrer Praxis nicht vorstellen können

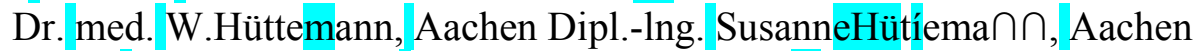

Privatliquidation - Praxiscomputeroder Verrech hungsstelle

PersÖnlichesZiel-,Zeit- u. Informations-Management,

praktischeTrps füreffizientes Arbeiten

- mitundohne PC -

Dipl.-Kfm. 0. Henker, Reutlingen 242

Praxis-EDV - MòglichkeitenderKommuníkation

Gudrun Demnick-Klas, Wöllstadt D.Fricke, Hannover

Praxiscomputer -VorgehensweisebeiEntscheidung, Auswahl und Einsatz

W.M. Lamers, Billerbeck

W. M. Lamers, Billerbeck

MKC (Messe-K $\beta t^{5} / 8$ gress-Ceniei'í Ost

Aktuelle AspektemodernerSchmerztherapie

DieMa aager-Ko $\pi$ ferenz -Erfolgsplanung.

Erfolgskontrolle, Steuerstrategien

Dr. G.Schlicht, Mannheim, Dipl.-Kfm. M. Bender, Jülich

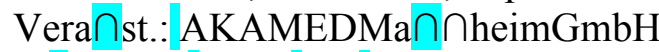

3

Prävention durch den Hausarzt Checkup - \$25 GabrielePrahi, Mannheim Veranst.

:AKAMEDManกheimGmbH

314

Prof. Dr. M.Zimmerman $\cap$, Heidelberg

BIOTEC

NeuíotropheimdnootropeFaktorenmForschung undMedizin

Prof. Dr. J. P. Huston, Düsseldorf 
Verhalíenstherapeutische Diagnostik und Therapie von Angst, Phobieu. Depression in derärztlichen Praxis

BIOTEC

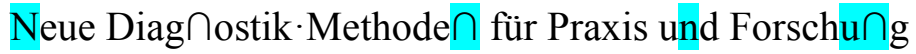

Diagnostik und Therapie der

- Refluxösophagitis - Non Ulcer Dysepsia - Nahrungs

mittelallergie - haemorrhagische Läsionen -

Prof.Dr.H.Brunner,F PDDr.W.K!ietmarin, Moers

PD Dr. E.KIieser, Düsseldorf Dr. W. StrauS, Düsseldorf

StateoftheArt

Dr.H.J.Hutt,Biberach

ro

Kòrper-und Ohrakupunktur in der täglichen Praxis

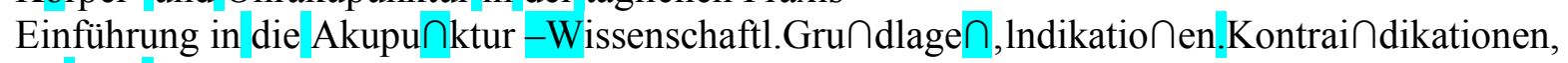
die Meridiane 